\title{
Emergence of a common energy scale close to the orbital-selective Mott transition
}

\author{
Markus Greger, Marcus Kollar, and Dieter Vollhardt \\ Theoretical Physics III, Center for Electronic Correlations and Magnetism, \\ Institute of Physics, University of Augsburg, 86135 Augsburg, Germany
}

(Dated: May 25, 2012)

\begin{abstract}
We calculate the spectra and spin susceptibilities of a Hubbard model with two bands having different bandwidths but the same on-site interaction, with parameters close to the orbital-selective Mott transition, using dynamical mean-field theory. If the Hund's rule coupling is sufficiently strong, one common energy scale emerges which characterizes both the location of kinks in the self-energy and extrema of the diagonal spin susceptibilities. A physical explanation of this energy scale is derived from a Kondo-type model. We infer that for multi-band systems local spin dynamics rather than spectral functions determine the location of kinks in the effective band structure.
\end{abstract}

PACS numbers: 71.27.+a, 71.30.+h

The interactions in correlated metals lead to the emergence of characteristic energy scales. Close to the Fermi energy Landau Fermi-liquid theory [1] applies and the effective electronic dispersion $E_{k}$ is renormalized, but remains linear as in the noninteracting case. The linear dependence terminates at an excitation energy which cannot be calculated within Fermi-liquid theory itself. With increasing strength of the electron-electron interaction this Fermi-liquid coherence scale decreases and ultimately vanishes at the Mott transition from a metal to an insulator. At the same time charge excitations are shifted to higher energies of the order of the interaction energy and are thus suppressed. For the single-band Hubbard model with on-site Coulomb interaction $U$ it was shown 2 in dynamical mean-field theory (DMFT) 3 that the Fermi-liquid regime terminates at an energy scale $\omega_{\Sigma}$ at which the real part of the selfenergy, $\operatorname{Re}[\Sigma(\omega)]$, and hence the effective dispersion $E_{\boldsymbol{k}}$, has a rather sudden change in slope 4. This 'kink' does not require any coupling to external bosonic degrees of freedom but is due to the correlated behavior of interacting electrons. For the single-band model the Fermi-liquid scale $\omega_{\Sigma}$ can be derived from the low-energy properties of the local spectral function 2. Moreover, it was demonstrated [5, 6] that the energy scale $\omega_{\Sigma}$ is linked to the characteristic energy scale $\omega_{\mathrm{sp}}$ of spin fluctuations. Kinks in the electronic dispersion were studied theoretically in a variety of contexts [7-14].

In this Letter we explore the origin and characteristic energy scale of kinks in the effective electronic dispersion in a more general context. Employing the DMFT we study a two-band Hubbard model with two different bandwidths, the same on-site repulsion $U$ for both bands, and an interorbital repulsion $U_{1}$ and ferromagnetic Hund's rule spin exchange $J$ between the bands. Thereby it is possible to capture orbital effects in correlated materials that do not exist in single-band models. Indeed, different kinks in the dispersion depending on the orbital character are observed for $\mathrm{Sr}_{2} \mathrm{RuO}_{4}$ both experimentally and theoretically [15, 16].
We study the model Hamiltonian

$$
\begin{aligned}
H= & \sum_{i j m \sigma} t_{i j, m} d_{i m \sigma}^{\dagger} d_{j m \sigma}+H_{\mathrm{int}} \\
H_{\mathrm{int}}= & U \sum_{i m} n_{i m \uparrow} n_{i m \downarrow}+\sum_{i \sigma \sigma^{\prime}}\left(U_{1}-\delta_{\sigma \sigma^{\prime}} J\right) n_{i 1 \sigma} n_{i 2 \sigma^{\prime}} \\
& +\frac{J}{2} \sum_{i m \sigma} d_{i m \sigma}^{\dagger}\left(d_{i \bar{m} \bar{\sigma}}^{\dagger} d_{i m \bar{\sigma}}+d_{i m \bar{\sigma}}^{\dagger} d_{i \bar{m} \bar{\sigma}}\right) d_{i \bar{m} \sigma},
\end{aligned}
$$

with spin index $\sigma=\uparrow, \downarrow$ and orbital index $m=1,2$. Here a bar over an index denotes the opposite spin or orbital. The two bands do not hybridize but are coupled by the interorbital interactions $U_{1}$ and $J$. We consider $U, U_{1}$ and $J$ as independent parameters which can take arbitrary values, but $U_{1}=U-2 J$ [17] for $d$ electrons. As in the single-band case the correlation strengths of the two bands may be roughly parametrized by the ratios $U / W_{1}$ and $U / W_{2}$, which are assumed to be unequal. Due to this difference in the relative interaction strengths an orbital-selective Mott transition (OSMT) occurs upon increase of $U$ [18 33]. We assume semi-elliptic densities of states, $\rho_{m}(\epsilon)=(8 / \pi) \sqrt{\left(W_{m} / 2\right)^{2}-\epsilon^{2}} / W_{m}^{2}$, with bandwidths $W_{1}<W_{2}$. Since the hopping amplitudes $t_{i j, m}$ are diagonal in the band index, so are the single-particle Green functions and self-energies. Off-diagonal contributions only occur in two-particle and higher-order correlation functions, e.g., spin and charge susceptibilities.

In DMFT the model (1) is mapped onto the following two-impurity Anderson model (TIAM) [28, 33.

$$
\begin{aligned}
H_{\mathrm{TIAM}}= & \sum_{\boldsymbol{k} m \sigma} \epsilon_{\boldsymbol{k} m} c_{\boldsymbol{k} m \sigma}^{\dagger} c_{\boldsymbol{k} m \sigma}+\sum_{m \sigma} \epsilon_{m} n_{m \sigma} \\
& +\sum_{\boldsymbol{k} m \sigma}\left(V_{\boldsymbol{k} m} c_{\boldsymbol{k} m \sigma}^{\dagger} d_{m \sigma}+\text { h.c. }\right)+H_{\mathrm{int}}^{\mathrm{loc}},
\end{aligned}
$$

where the local interaction $H_{\text {int }}^{\text {loc }}$ has the same form as $H_{\text {int }}$, but without the index $i$. The DMFT selfconsistency conditions demand that the band energies $\epsilon_{\boldsymbol{k} m}$ and hybridizations $V_{\boldsymbol{k} m}$ are determined such that Green functions and self-energies of (2) equal the corre- 

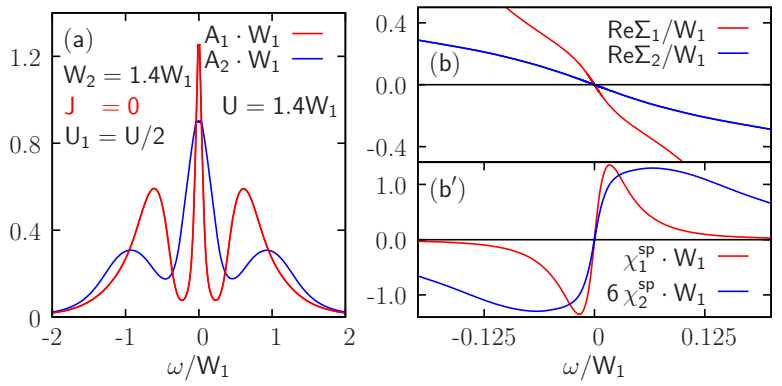

FIG. 1. For $J=0$ and $U_{1} \neq 0$ the difference in the correlation strength of the two bands is observed not only in the shape of the spectral function $A_{m}(\omega)$ (a), but also in the corresponding band-resolved Kondo temperatures and widths of the Fermiliquid regime $\left(\mathrm{b}, \mathrm{b}^{\prime}\right)$.

sponding local lattice quantities,

$$
\begin{aligned}
G_{m}(\omega) & =\int d \epsilon \frac{\rho_{m}(\epsilon)}{\omega+i 0-\epsilon_{m}-\Sigma_{m}(\omega)-\epsilon}, \\
\Sigma_{m \sigma}(\omega) & =\omega+i 0-\epsilon_{m}-\frac{1}{G_{m}(\omega)}-\Delta_{m}(\omega),
\end{aligned}
$$

which take the same form as for two decoupled oneband models due to the absence of interorbital hopping. Here the hybridization function is defined as $\Delta_{m}(\omega)=$ $\sum_{\boldsymbol{k}}\left|V_{\boldsymbol{k} m}\right|^{2} /\left(\omega+i 0-\epsilon_{\boldsymbol{k} m}\right)$. We solve the impurity model using the Numerical Renormalization Group (NRG). The complete Fock space NRG 34 proceeds as in the singleband Hubbard model 35, but with a local dimension of 16 for the impurity and the chain sites [19, 35. Unless noted otherwise the NRG discretization parameter is $\Lambda=2.5$, and we keep on the order of $10^{5}$ states, including multiplicities of irreducible subspaces, in each NRG iteration. Although we focus on the low-energy region we employ Oliveira's 36] $z$-trick $\left(N_{z}=4\right)$ to improve the spectra at higher energies. To obtain the selfenergy with high quality the correlation function $F_{m}(\omega)$ $=\left\langle\left\langle\left[d_{m, \sigma}, H_{\mathrm{int}}^{\text {loc }}\right] ; d_{m, \sigma}^{\dagger}\right\rangle_{\omega}\right.$ is calculated since it is numerically better conditioned than the Dyson equation [37.

We compute the spectra, self-energies, and spin susceptibilities for the two bands in the metallic phase close to the OSMT, and monitor the behavior as a function of the Hund's rule coupling $J$. The different correlation strengths of the orbitals lead to different behavior of the spectral functions $A_{m}(\omega)=-\operatorname{Im}\left[G_{m}(\omega)\right] / \pi$ and selfenergies $\Sigma_{m}(\omega)$ which are shown in Figs. 1 and 2. The overall behavior corresponds to that of two Fermi liquids with different mass renormalizations, reminiscent of two uncoupled one-band Hubbard models with different local interactions. In all cases the single-particle spectra $A_{1}(\omega)$ and $A_{2}(\omega)$ differ significantly, especially close to the OSMT when the spectral function of the narrow band has a very sharp central peak. Because the spectrum of each band depends mostly on the correlation strength $U / W_{m}$ but not much on Hund's rule exchange
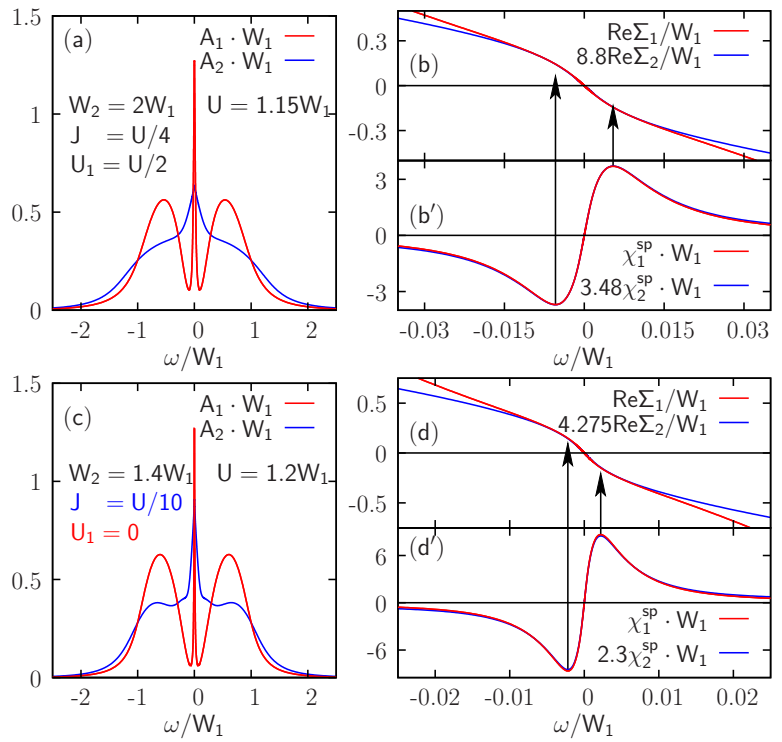

FIG. 2. In the metallic phase close to the OSMT a striking proportionality, $\operatorname{Re}\left[\Sigma_{1}\right] \propto \operatorname{Re}\left[\Sigma_{2}\right]$ and $\chi_{1}^{\mathrm{sp}} \propto \chi_{2}^{\mathrm{sp}}$ (panels b, $\mathrm{b}^{\prime}, \mathrm{d}, \mathrm{d}^{\prime}$ ) is seen to emerge for $J>0$, which is in marked contrast to the decoupled behavior in Fig. 1. Very close to the OSMT $\left(\mathrm{d}, \mathrm{d}^{\prime}\right)$ even a weak exchange coupling $J$ leads to a common low-energy scale. Note that $U_{1}$ has little influence on the qualitative low-energy behavior.

$J$, the latter was previously characterized as a 'band decoupler' 26], at least for weak interorbital hopping. Quantum-Monte Carlo results [21, 23, 25, 28, 29] suggest that at $T=0$ the low-frequency behavior of $\operatorname{Re}\left[\Sigma_{m}\right]$ and $\chi_{m}^{\mathrm{sp}}$ is then also different. Fig. 1 shows that this is indeed the case - but only for $J=0$ and $U_{1} \neq 0$. Indeed, a finite Hund's rule coupling leads to a fundamentally different low-energy behavior of $\operatorname{Re}\left[\Sigma_{m}\right]$ and $\chi_{m}^{\text {sp }}$. Namely, as the system approaches the OSMT we find that at low energies these quantities become proportional, i.e., $\operatorname{Re}\left[\Sigma_{1}(\omega)\right] \propto \operatorname{Re}\left[\Sigma_{2}(\omega)\right]$ and $\chi_{1}^{\mathrm{sp}}(\omega) \propto \chi_{2}^{\mathrm{sp}}(\omega)[38$. As illustrated in Figs. 2b, $2 \mathrm{~b}^{\prime}, 2 \mathrm{~d}, 2 \mathrm{~d}^{\prime}$ this striking result cannot be inferred from the spectral functions $A_{m}(\omega)$ since the shape and the characteristic energy scales of the latter differ considerably and thus suggest a decoupled behavior. The characteristic energy scale of the spin fluctuations, i.e., the locations $\omega_{m}^{\mathrm{sp}}$ of the extrema in the spin susceptibilities $\chi_{m}^{\mathrm{sp}}(\omega)$, allow one to define a Kondo temperature for each band. The proportionalities discussed above imply that the system has identical Kondo temperatures $\left(\omega_{1}^{\mathrm{sp}}=\omega_{2}^{\mathrm{sp}}\right)$, Fermi-liquid energy scales and kinks $\left(\omega_{1}^{\Sigma}=\omega_{2}^{\Sigma}\right)$, irrespective of the different correlation strengths of the bands. Furthermore, the self-energy kinks and the strongest spin fluctuations occur at the same energy in each band, $\omega_{m}^{\Sigma} \simeq \omega_{m}^{\mathrm{sp}}$ (as observed also in the single-band case [5]), which means that for the two-band model (1) a single common low-energy scale emerges for kinks and spin fluctuations in both bands. For our numerical data we define the kink scale $\omega_{m}^{\Sigma}$ as 


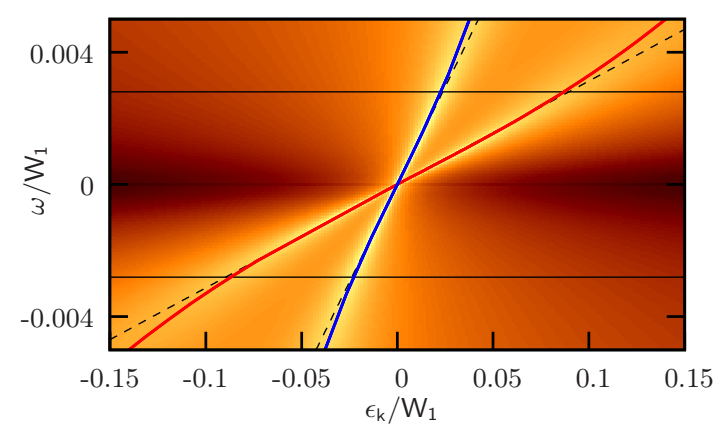

FIG. 3. Intensity plot of the total momentum-resolved spectral function $A_{\boldsymbol{k}}(\omega)$ deep inside the central peaks as a function of $\epsilon_{\boldsymbol{k}}$ and $\omega$ for the same parameters as in Fig. $2 \mathrm{p}$, b. The effective dispersion $E_{\boldsymbol{k}, m}$ is given by the local maxima in $A_{\boldsymbol{k}}(\omega)$ (blue line for the wide band, lighter red line for the narrow band). It is linear (dashed lines) in the Fermi-liquid regime close to the Fermi surface and has kinks at the same energy $\sim \pm 0.0028 W_{1}$ for both bands (solid horizontal lines).

the energy for which the extrapolated linear dispersion near the Fermi energy deviates from $\operatorname{Re}\left[\Sigma_{m}(\omega)\right]$ by $20 \%$, which agrees well with the perceived location of the kinks in Figs. 2 $2 \mathrm{~b}, \mathrm{~d}$. The corresponding momentum-resolved spectral function $A_{\boldsymbol{k}}(\omega)$ and effective dispersions $E_{\boldsymbol{k} m}$ are shown Fig. 3 . We observe that although the slope of the Fermi-liquid dispersion is very different for the two bands, the linear regimes terminate at the same energy scale, which however slightly deviates from $\omega_{m}^{\Sigma}$ due to band structure effects.

By comparing the results for $J=0$ in Fig. 1 with $J \neq 0$ in Figs. 2 b-c it is clear that the interorbital repulsion $U_{1}$ is not responsible for the common energy scale. This effect only appears in the presence of the Hund's rule coupling $J$, whereas $U_{1}$ merely leads to quantitative modifications. We will thus restrict ourselves to $U_{1}=0$ in the following. Starting from $J=0$ we study the continuous evolution of the two initially uncoupled Hubbard models into the 'locked' regime. To this end we obtain Kondo scales $\omega_{m}^{\mathrm{sp}}(J)$ and self-energy kinks $\omega_{m}^{\Sigma}(J)$ for the two orbitals for two different values of $U / W_{1}$ (Figs. 4a $-\mathrm{b}$ ). As $J$ is increased both orbital-resolved energy scales approach each other and finally merge into a single scale, as seen in Figs. 2 $2 k-d^{\prime}$. Comparing Figs. 4 4 and b, we observe that this common low-energy scale appears at a threshold value which decreases for increasing $U$. We also notice the very close correspondence between kink energies and Kondo-temperatures, especially in the more strongly correlated case (Fig. 4b). This observation can be understood in terms of local Fermi-liquid theory [39]: Since the binding energy of the Kondo singlet is approximately given by the Kondo temperature, the linear regime must terminate at $\omega \simeq \omega_{\mathrm{sp}}$; see Refs. [5, 6] for a discussion of the single-band case. As expected the kink energy scale derived in Ref. [2], $\omega_{m}^{\star}=0.2 Z_{m} W_{m}$, applies only to the narrow band with its well-developed three-peak
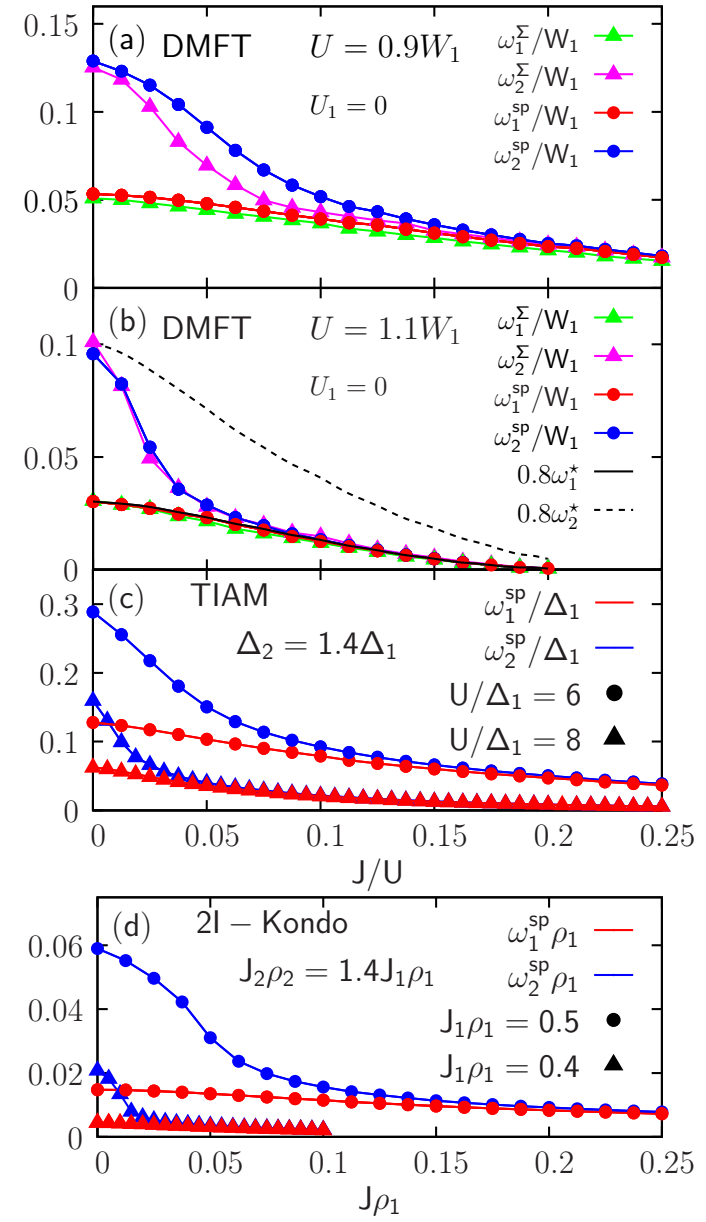

FIG. 4. (a,b) Orbitally resolved kink energies $\omega_{1,2}^{\Sigma}(J)$ and Kondo temperatures $\omega_{1,2}^{\mathrm{sp}}(J)$ as calculated within DMFT for different Hubbard interactions $U$. Sufficiently large $U$ leads to $\omega_{m}^{\Sigma}(J)=\omega_{m}^{\mathrm{sp}}(J)$ and sufficiently large $J$ leads to $\omega_{1}^{\Sigma}(J)=$ $\omega_{2}^{\Sigma}(J)$ and $\omega_{1}^{\mathrm{sp}}(J)=\omega_{2}^{\mathrm{sp}}(J)$. The dashed lines in (b) mark the single-band estimate for $\omega_{m}^{\Sigma}$ 2] which applies only to the narrow band (see text). (c) A simplified Anderson impurity model and, (d), a related two-impurity Kondo model, both with a behavior similar to the DMFT solution. Note that the system in (b) enters the OSMT phase at approximately $J=0.2 U$. For these very low-energy features $\mathcal{O}(5500)$ states were kept and $N_{z}=2$.

spectral function and not to the wide band (cf. solid and dashed lines, respectively, in Fig. 4 $\mathrm{b}$, corresponding to $0.8 \omega_{m}^{\star}[40]$.

In the vicinity of the OSMT the characteristic energies $\omega_{m}^{\mathrm{sp}}(J)$ and $\omega_{m}^{\Sigma}(J)$ represent equivalent energy scales and hence contain the same physical information. In order to explain the $J$ dependence it suffices to discuss one of them, and we will focus on $\omega_{m}^{\mathrm{sp}}(J)$ in the following. To explain the locking of the low-energy scales for the two bands we proceed in two steps; see Figs. 化 and $4 \mathrm{~d}$. First we establish that the locking is an intrinsic property of the underlying TIAM Hamiltonian and is only quantitatively modified by the DMFT self-consistency 
equations (3). Then we compare with the results for a Kondo-type model that allows us to identify the competing couplings and elementary excitations. For the first step we solve the impurity model (2) with different but constant hybridization functions $\left(\Delta_{2}(\omega)=1.4 \Delta_{1}(\omega)=\right.$ const) and extract the Kondo temperatures $\omega_{m}^{\mathrm{sp}}(J)$ from the maxima of the spin susceptibilities. The result is depicted in Fig. 4 for two values of $U$, showing very good qualitative agreement with the DMFT results in Figs. 4a, 4b. In particular, the common low-energy scale emerges at a value of $J$ which decreases with increasing $U$ in a similar fashion. We conclude that the DMFT selfconsistency induces only minor modifications as long as the system remains in the metallic phase.

In a second step we focus on the low-energy spin dynamics close to the OSMT. In this regime charge excitations are strongly suppressed. We thus consider the Kondo limit of (2) which captures the low-energy spin dynamics of the TIAM in Fig. 4. In this limit the Hamiltonian (2) reduces to a two-impurity Kondo model (2IKM) [41, 42],

$$
\begin{aligned}
H_{2 \mathrm{IKM}}=\sum_{\boldsymbol{k} m \sigma} \epsilon_{\boldsymbol{k} m} c_{\boldsymbol{k} m \sigma}^{\dagger} c_{\boldsymbol{k} m \sigma} & \\
& +\sum_{m} J_{m} \boldsymbol{s}_{m} \cdot \boldsymbol{S}_{m}-J \boldsymbol{S}_{1} \cdot \boldsymbol{S}_{2} .
\end{aligned}
$$

Here $J>0$ is the Hund's exchange interaction of (2), while the antiferromagnetic couplings $J_{m}$ stem from superexchange processes and decrease with increasing $U$. We take $J_{2} \rho_{2}(0)=1.4 J_{1} \rho_{1}(0)$ to obtain different Kondo temperatures for $J=0$, i.e., $\omega_{1}^{\mathrm{sp}}(0) \neq \omega_{2}^{\mathrm{sp}}(0)$. The $J$ dependence of $\omega_{m}^{\mathrm{sp}}(J)$ is shown in Fig. $4 \mathrm{~d}$. The qualitative agreement among the results obtained for all three models (DMFT, TIAM with constant hybridization, 2IKM) confirms that (4) already describes the essential processes that lead to the emergence of the joint low-energy scale. In the 2IKM the spins will align for low-excitation energies and form a composite spin-1 object [41. This happens roughly when the energy gain $\omega_{1}^{\mathrm{sp}}(J)+\omega_{2}^{\mathrm{sp}}(J)$ due to Kondo screening of the two impurities is overcome by $J / 4$, the approximate energy gain due to the ferromagnetic exchange. Hence the locking of the low-energy scales sets in at about $J \approx \omega_{m}^{\mathrm{sp}}(J) / 8$, as seen in Fig. 4a $\left(J \approx 0.2 W_{1}\right)$ and $4 \mathrm{~b}\left(J \approx 0.1 W_{1}\right)$. We conclude that the low-energy spin dynamics of the two impurity spins (and thus the two bands of the corresponding lattice model in DMFT) exhibit joint fluctuations and thus have equal Kondo scales if $J$ dominates the individual Kondo scales $\omega_{m}^{\mathrm{sp}}$ and are essentially independent otherwise. Regarding the influence of $U$, we note that the antiferromagnetic couplings between the spins and the baths decrease with increasing interaction, i.e., more correlated systems exhibit stronger locking of their spins and their low-energy scales. Thus $J$ couples the low-energy scales more effectively for stronger correlations, as seen in Fig. 4 a-c.

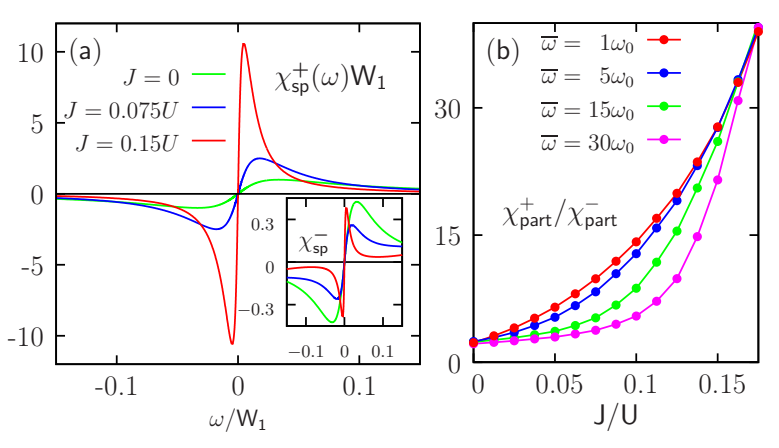

FIG. 5. The correlation functions $\chi_{\mathrm{sp}}^{+}(\omega)$ and $\chi_{\mathrm{sp}}^{-}(\omega)$ indicate the creation of a composite spin-1 object for large $J$ (green curves in (a) and in the inset have about the same magnitude; also note the different scales in (a) and the inset). Accordingly, the partial spectral weight fraction $\chi_{\text {part. }}^{+} / \chi_{\text {part. }}^{-}$in (b) grows strongly with $J$ and is only quantitatively affected by the upper limit $\bar{\omega}$. Here $\omega_{0}=\max \left[\omega_{1}^{\mathrm{sp}}(J), \omega_{2}^{\mathrm{sp}}(J)\right]$.

To explicitly verify the physical picture described above we investigate the correlation functions

$$
\chi_{\mathrm{sp}}^{ \pm}(\omega)=\left\langle\left\langle\boldsymbol{S}_{1} \pm \boldsymbol{S}_{2} \mid \boldsymbol{S}_{1} \pm \boldsymbol{S}_{2}\right\rangle\right\rangle_{\omega}
$$

in DMFT. They describe the dynamics of the composite spin-1 object $\chi^{+}(\omega)$ and the 'residual' singlet $\chi^{-}(\omega)$, respectively, and are plotted in Fig. 5a. As expected, for both susceptibilities the positions of their maxima decreases with increasing interaction. However, only the composite spin- 1 object shows a resonance that also increases in amplitude. In Fig. 5b we plot the integrated weight $\chi_{\text {part }}^{ \pm}=\int_{0}^{\bar{\omega}} \chi^{ \pm}(\omega) d \omega$ as a function $J$ for several values of the upper limit $\bar{\omega}$. In particular, for larger values of $J$ the residual spin contributes only little to the total low-energy spin response $\chi_{1}^{\mathrm{sp}}+\chi_{2}^{\mathrm{sp}}$ of the system, which is thus well described by $\chi^{+}(\omega)$. This establishes the formation of the composite spin- 1 object as the physical origin for the emergence of the common energy scale. Namely, as the OSMT is approached by increasing $U$, the antiferromagnetic superexchange of the narrow and wide band both decrease until $J$ becomes the dominating scale, at least for the spins in the narrow band. The spins align and create a composite spin-1 and exhibit joint low-energy dynamics, leading to the proportionalities of $\operatorname{Re}\left[\Sigma_{m}(\omega)\right]$ and $\chi_{m}^{\mathrm{sp}}(\omega)$ for the two bands.

In summary, we explored the physical mechanism for kinks responsible for the appearance of kinks in the self-energy by studying a two-band model with different bandwidths but the same local charge interactions as well as Hund's rule spin exchange. We find that the physical picture developed previously for single-band systems close to the Mott transition is significantly modified for strong Hund's rule coupling, due to the formation of a local spin-1 object. As a consequence, a common lowenergy scale emerges for the two bands for the kinks in the self-energies and the maxima in the spin susceptibilities. 
We thank R. Žitko for releasing his NRG code, on which our code is based [43, and L. Chioncel and J. Otsuki for useful discussions. This work was supported by the Deutsche Forschungsgemeinschaft through TRR 80.

[1] D. Pines and P. Nozières, The Theory of Quantum Liquids, Vol. I (Benjamin, New York, 1966).

[2] K. Byczuk, M. Kollar, K. Held, Y.-F. Yang, I. A. Nekrasov, T. Pruschke, and D. Vollhardt, Nature Physics 3, 168 (2007).

[3] W. Metzner and D. Vollhardt, Phys. Rev. Lett. 62, 324 (1989) A. Georges, G. Kotliar, W. Krauth, and M. J. Rozenberg, Rev. Mod. Phys. 68, 13 (1996), G. Kotliar and D. Vollhardt, Physics Today 57, 53 (2004).

[4] An effective dispersion $E_{k}$ can always be defined by the maxima of the momentum-resolved spectral function $A_{k}(\omega)$, which is essentially measured in angular-resolved photoemission spectroscopy (ARPES), even when the spectrum is rather incoherent and no sharp quasiparticle excitations occur.

[5] C. Raas, P. Grete, and G. S. Uhrig, Phys. Rev. Lett. 102, 076406 (2009)

[6] P. Grete, S. Schmitt, C. Raas, F. B. Anders, and G. S. Uhrig, Phys. Rev. B 84, 205104 (2011)

[7] M. Eschrig and M. R. Norman, Phys. Rev. Lett. 85, 3261 (2000).

8] D. Manske, I. Eremin, and K. H. Bennemann, Phys. Rev. Lett. 87, 177005 (2001)

[9] Y. Kakehashi and P. Fulde, J. Phys. Soc. Jpn. 74, 2397 (2005)

|10| I. A. Nekrasov, K. Held, G. Keller, D. E. Kondakov, T. Pruschke, M. Kollar, O. K. Andersen, V. I. Anisimov, and D. Vollhardt, Phys. Rev. B 73, 155112 (2006)

[11] A. Toschi, M. Capone, C. Castellani, and K. Held, Phys. Rev. Lett. 102, 076402 (2009)

[12] A. Hofmann, X. Y. Cui, J. Schäfer, S. Meyer, P. Höpfner, C. Blumenstein, M. Paul, L. Patthey, E. Rotenberg, J. Bünemann, F. Gebhard, T. Ohm, W. Weber, and R. Claessen, Phys. Rev. Lett. 102, 187204 (2009).

[13] X. Deng, M. Ferrero, J. Mravlje, M. Aichhorn, and A. Georges, Phys. Rev. B 85, 125137 (2012).

[14] J. Bauer and G. Sangiovanni, Phys. Rev. B 82, 184535 (2010)

[15] H. Iwasawa, Y. Aiura, T. Saitoh, I. Hase, S. I. Ikeda, Y. Yoshida, H. Bando, M. Higashiguchi, Y. Miura, X. Y. Cui, K. Shimada, H. Namatame, and M. Taniguchi, Phys. Rev. B 72, 104514 (2005)

[16] J. Mravlje, M. Aichhorn, T. Miyake, K. Haule, G. Kotliar, and A. Georges, Phys. Rev. Lett. 106, 096401 (2011)

[17] C. Lyon-Caen and M. Cyrot, J. Phys. C: Sol. St. Phys. 8, $2091(1975)$
[18] V. Anisimov, I. Nekrasov, D. Kondakov, T. Rice, and M. Sigrist, Eur. Phys. J. B 25, 191 (2002).

[19] T. Pruschke and R. Bulla, Eur. Phys. J. B 44, 217 (2005)

[20] A. Liebsch, Phys. Rev. Lett. 95, 116402 (2005) T. A. Costi and A. Liebsch, Phys. Rev. Lett. 99, 236404 (2007)

[21] C. Knecht, N. Blümer, and P. G. J. van Dongen, Phys. Rev. B 72, 081103 (2005)

[22] E. Jakobi, N. Blümer, and P. G. J. van Dongen, Phys. Rev. B 80, 115109 (2009).

23 R. Arita and K. Held, Phys. Rev. B 72, 201102 (2005).

[24] S. Biermann, L. de'Medici, and A. Georges, Phys. Rev. Lett. 95, 206401 (2005)

25] L. de'Medici, A. Georges, and S. Biermann, Phys. Rev. B 72, 205124 (2005)

[26] L. de'Medici, Phys. Rev. B 83, 205112 (2011)

[27] A. Koga, N. Kawakami, T. M. Rice, and M. Sigrist, Phys. Rev. Lett. 92, 216402 (2004), K. Inaba, A. Koga, S. Suga, and N. Kawakami, Phys. Rev. B 72, 085112 (2005).

[28] A. Koga, N. Kawakami, T. Rice, and M. Sigrist, Physica B: Cond. Matter 359-361, 1366 (2005).

[29] K. Bouadim, G. G. Batrouni, and R. T. Scalettar, Phys. Rev. Lett. 102, 226402 (2009)

[30] J. Bünemann, D. Rasch, and F. Gebhard, J. Phys.: Cond. Matter 19, 436206 (2007).

[31] R. Peters and T. Pruschke, Phys. Rev. B 81, 035112 (2010)

[32] W.-C. Lee and P. W. Phillips, Phys. Rev. B 84, 115101 (2011)

|33| L. de'Medici, J. Mravlje, and A. Georges, Phys. Rev. Lett. 107, 256401 (2011) A. Georges, Ann. Phys. 523, 672 (2011) K. Inaba and A. Koga, Phys. Rev. B 73, $155106(2006)$

[34] R. Peters, T. Pruschke, and F. B. Anders, Phys. Rev. B 74, 245114 (2006)

[35] R. Bulla, Phys. Rev. Lett. 83, 136 (1999)

[36] W. C. Oliveira and L. N. Oliveira, Phys. Rev. B 49, 11986 (1994)

[37] R. Bulla, A. C. Hewson, and T. Pruschke, J. Phys.: Cond. Matter 10, 8365 (1998).

[38] A similar proportionality is found for the imaginary part of the self-energy, $\operatorname{Im}\left[\Sigma_{1}(\omega)\right] \propto \operatorname{Im}\left[\Sigma_{2}(\omega)\right]$, in the same interval as for its real part.

[39] P. Nozières, J. Low Temp. Phys. 17, 31 (1974)

[40] The prefactor of 0.8 accounts for our present kink criterion $(20 \%$ deviation in the self-energy from the linear extrapolation, see text), which differs slightly from that in Ref. 2].

[41] C. Jayaprakash, H. R. K. Murthy, and J. W. Wilkins, Phys. Rev. Lett. 47, 737 (1981).

[42] B. A. Jones and C. M. Varma, Phys. Rev. Lett. 58, 843 (1987)

[43] R. Žitko, Comp. Phys. Comm. 182, 2259 (2011), http: //nrgljubljana.ijs.si 